\title{
Multidimensional Pulse-Position Coded-Modulation for Deep-Space Optical Communication
}

\author{
Ivan B. Djordjevic, Senior Member, IEEE
}

\begin{abstract}
In order to achieve multigigabit transmission (projected for 2020) for the use in interplanetary communications, in this letter, we propose the use of multidimensional pulse-position modulation (PPM). From Shannon's theory, we know that information capacity is a logarithmic function of signal-to-noise ratio, but a linear function of number of dimensions. By using pulse-positions as a basis function, we can improve the spectral efficiency of conventional PPM. The $N$-dimensional PPM (ND-PPM) can, therefore, be used to solve the high-bandwidth requirements of future deep-space optical communications. The $N$-dimensional signal constellation can be obtained as $N$-dimensional Cartesian product one-dimensional pulse-amplitude modulation constellation. The improvement of ND-PPM over PPM for $N=8$ in strong turbulence regime is even $3.21 \mathrm{~dB}$ at a bit-error rate (BER) of $10^{-5}$. In addition, the spectral efficiency of the proposed scheme is $N / \log _{2} N$ times better than that of PPM.
\end{abstract}

Index Terms-Atmospheric turbulence, coded modulation, deep-space optical communication, low-density parity-check (LDPC) codes, multidimensional pulse-position modulation.

\section{INTRODUCTION}

$\mathbf{P}$ OWER-EFFICIENT modulation schemes, such as pulseposition modulation (PPM), are widely adopted in current deep-space optical communications [1]-[4]. The very large bandwidth of these links (compared to RF links) has made the low spectral efficiency of PPM less of a concern. However, in order to achieve multigigabit transmission (projected for 2020) for the use in interplanetary communications [4], the usage of large number of time slots in PPM is needed, which imposes stringent requirements on system design and implementation. On the other hand, from Shannon's theory we know that information capacity is a linear function of number of dimensions, and a logarithmic function of signal-to-noise ratio. Therefore, by increasing the number of dimensions we can dramatically improve the overall data rate.

In this letter, we propose the use of large-girth regular lowdensity parity-check (LDPC) coded $N$-dimensional pulse-position modulation (ND-PPM) as next generation of deep-space optical communication enabling technology. This scheme can also be called the generalized PPM. We show by Monte Carlo simulation for Poisson deep-space optical channel model, that improvement of ND-PPM over PPM ranges from $0.69 \mathrm{~dB}$ for

Manuscript received April 21, 2011; revised June 17, 2011; accepted June 24, 2011. Date of publication June 30, 2011; date of current version August 31, 2011. This letter was supported in part by the NSF under Grant CCF-0952711, Grant ECCS-0725405, and Grant EEC-0812072.

The author is with the ECE Department, University of Arizona, Tucson, AZ 85721 USA (e-mail: ivan@email.arizona.edu).

Color versions of one or more of the figures in this letter are available online at $\mathrm{http}: / /$ ieeexplore.ieee.org.

Digital Object Identifier 10.1109/LPT.2011.2160940
$N=2$ to $2.83 \mathrm{~dB}$ for $N=8$ at bit-error rate (BER) of $10^{-5}$, for average number of background photons $n_{b c}$ of 0.1 . Under strong atmospheric turbulence, the improvement is even better (3.21 dB at the same BER for $N=8$ ). Moreover, the spectral efficiency of proposed scheme, for two amplitude levels per dimension, is $\mathrm{N} / \log _{2} N$ times better than that of PPM. The proposed scheme is more flexible as it can be used for arbitrary number of dimensions (slots), while PPM can be used only for number of slots being power of two.

\section{DeEP-Space Optical Communications Based on LDPC-CODED ND-PPM}

In this section, we provide a detailed description of our proposed $N$-dimensional pulse-position modulation (ND-PPM) approach to enable ultra-high-speed deep-space optical communication. In proposed scheme, we employ $N$ pulse-position basis functions defined as follows:

$$
\Phi_{j}(t)=\frac{1}{\sqrt{T_{s} / N}} \operatorname{rect}\left[\frac{t-(j-1) T_{s} / N}{T_{s} / N}\right] ; j=1, \ldots, N
$$

where $T_{s}$ is a symbol duration, and $\operatorname{rect}(t)$ is defined as

$$
\operatorname{rect}(t)=\left\{\begin{array}{ll}
1, & 0 \leq t<1 \\
0, & \text { otherwise }
\end{array} .\right.
$$

The overall system configuration is shown in Fig. 1. The key difference between PPM and our proposal is that PPM is an orthogonal signaling scheme (symbols are mutually orthogonal), while the proposed scheme is not orthogonal. Namely, the proposed scheme employs the pulse-positions as basis functions (coordinates), multiple nonzero components per symbol can exist, indicating that the symbols are not orthogonal among each other. Note that arbitrary basis functions can be used. However, the use of pulse-position basis functions is compatible with currently existing deep-space technologies. As depicted in the Fig. 1(a), $K$ different bit streams coming from different information sources are encoded using $K(n, k)$ LDPC encoders operating in parallel. Therefore, our scheme employs $K$ LDPC encoders/decoders operating in parallel at data rate $R_{s}\left(R_{s}\right.$ is the symbol rate), while [1] employs one LDPC encoder/decoder operating at data rate $K R_{s}$, which is more challenging in multiGiga symbols/s range. The outputs of the encoders are interleaved by the $K \times n$ block interleaver. The block interleaver accepts bits from the encoders row-wise and outputs bits columnwise to the mapper, which accepts $K$ bits at each symbol interval $i$. The mapper determines the corresponding $L^{N}$-ary signal constellation point by

$$
s_{i}=C_{N} \sum_{j=1}^{N} \varphi_{i, j} \Phi_{j} ; i=1,2, \ldots, L^{N}
$$



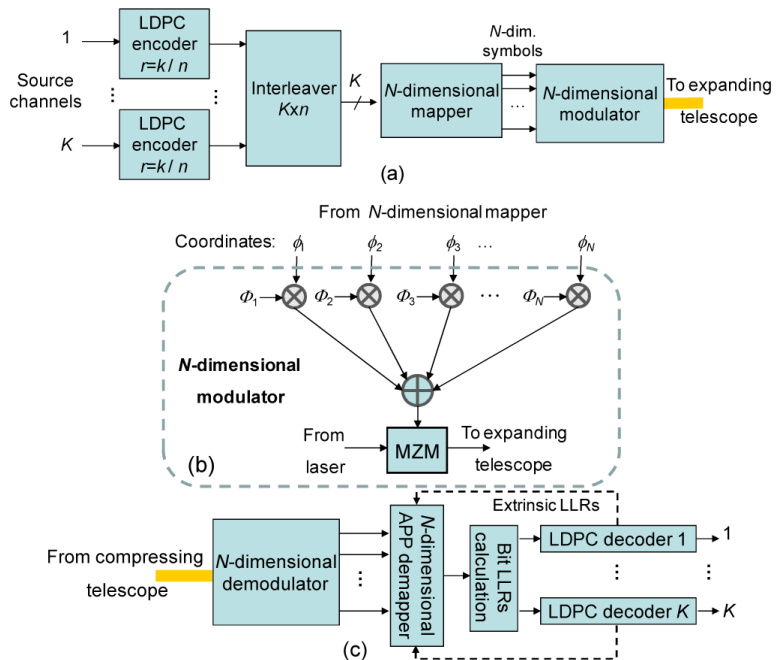

(c)

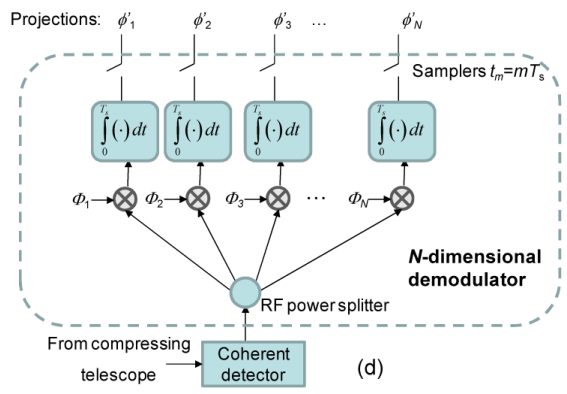

Fig. 1. LDPC-coded ND-PPM system: (a) transmitter configuration, (b) $N$-dimensional modulator configuration, (c) receiver configuration, and (d) $N$-dimensional coherent demodulator configuration. MZM: Mach-Zehnder modulator. APP: a posteriori probability demapper.

where $L$ is the number of amplitude levels per basis function and $C_{N}$ is the normalization constant. The set $\left\{\Phi_{1}, \Phi_{2}, \ldots, \Phi_{N}\right\}$ represents a set of $N$ orthogonal PPM basis functions given by (1), while with $\varphi_{i, j}$ we denoted ND-PPM coordinates of $i$ th constellation point. The signals are then combined, modulated, and sent towards remote deep-space destination by an expanding telescope (see Fig. 1). The number basis functions $N$ to be used is determined by the desired final rate and current channel conditions. The simplest ND-PPM coded modulation scheme with direct detection can be described by the following set of constellation points for $N=3$ and $L=2$ as $\quad\{(0,0,0),(0,0,1),(0,1,0),(0,1,1),(1,0,0),(1,0,1)$, $(1,1,0),(1,1,1)\}$. The $N$-dimensional signal constellation can be obtained as the $N$-dimensional Cartesian product of a one-dimensional signal constellation originating from nonnegative pulse-amplitude modulation (PAM). For coherent detection version zeros in signal constellation above need to be replaced by $-1 \mathrm{~s}$. One possible $N$-dimensional modulator configuration is shown in Fig. 1(b). The $N$-coordinates from mapper are used as inputs to the modulator, properly multiplied with corresponding basis functions (see (1), (2)), combined and after driver amplifier used as an RF input to the Mach-Zehnder modulator (MZM). Notice that the electrical portion of modulator can be implemented as a DSP module followed by a digital-to-analog converter (DAC). The modulated optical signal after MZM is amplified and with help of an expanding telescope send to a deep-space destination of interest.

At the receiver side, as shown in Fig. 1(c), after $N$-dimensional demodulation and photodetection, the outputs of the $N$ branches of the demodulator are sampled at the symbol rate and the corresponding samples are forwarded to a posteriori probability (APP) demapper. The $N$-dimensional demodulator can be implemented in many different ways. The coherent detection version is shown in Fig. 1(d). The coherent detector provides the $N$-dimensional signal used as input to the corresponding demodulator, depicted in Fig. 1(d). The outputs of correlators, from Fig. 1(d), provide projections along corresponding basis functions (given by (1)). Instead of the bank of correlators we can use a bank of matched filters. This particular version is suitable for DSP implementation. Namely, we can use an analog-to-digital conversion blocks and perform match filtering in digital domain followed by dot-product calculation among received vector and candidate constellation point vectors. Such obtained projections are used as inputs of corresponding APP demapper, as shown in Fig. 1(c). The direct detection version of $N$-dimensional receiver can be implemented by using a power splitter (1: $N$ star coupler), whose outputs are used as input to $N$-photodetectors/photon counter receivers which integrates the signal/performs photon counting over corresponding intervals of duration $T_{s} / N$ starting at $(n-1) T_{s} / N(n=1, \ldots, N)$, respectively. The corresponding samples from $N$ photodetectors/photon counters are passed to the APP demapper. The APP demapper provides the bit LLRs required for iterative LDPC decoding (see Fig. 1(c)). The $n$th branch in Fig. 1(d) represents the projection along $n$th basis function (coordinate). Let $S_{i}=\left[S_{i}^{(1)} \cdots S_{i}^{(N)}\right]^{T}$ denote the transmitted signal constellation point, $\boldsymbol{R}_{i}$ denote the received constellation point, and $S_{0}$ denote the reference constellation point. The superscript $(n)(n=1, \ldots, N)$ is used to denote the $n$th coordinate. Let $P\left(\boldsymbol{R}_{i} \mid \boldsymbol{S}_{i}\right)$ denote the conditional probabilities that depend on deep-space channel model (see [2] for various deep-space channel models, including Poisson channel model), while $P(\boldsymbol{S})$ denotes a priori probability of symbol $\boldsymbol{S}$. The symbol log-likelihood ratios (LLRs) can be calculated by

$$
\lambda\left(\boldsymbol{S}_{i}\right)=\log \left[P\left(\boldsymbol{S}_{i} \mid \boldsymbol{R}_{i}\right) / P\left(\boldsymbol{S}_{0} \mid \boldsymbol{R}_{i}\right)\right],
$$

where $P\left(\boldsymbol{S}_{i} \mid \boldsymbol{R}_{i}\right)$ is determined by Bayes' rule, and upon its application (3) becomes

$$
\lambda\left(\boldsymbol{S}_{i}\right)=\log \left[P\left(\boldsymbol{R}_{i} \mid \boldsymbol{S}_{i}\right) / P\left(\boldsymbol{R}_{i} \mid \boldsymbol{S}_{0}\right)\right]+\lambda_{a}\left(\boldsymbol{S}_{i}\right),
$$

where $\lambda_{a}\left(\boldsymbol{S}_{i}\right)=\log \left[P\left(\boldsymbol{S}_{i}\right) / P\left(\boldsymbol{S}_{0}\right)\right]$. The prior symbol estimate can be obtained from

$$
\lambda_{a}(\hat{s})=\sum_{j=1}^{b} c_{j} L_{D, e}\left(c_{j}\right),
$$

where

$$
L_{D, e}\left(\hat{c}_{j}\right)=L\left(c_{j}^{\text {(out) }}\right)-L\left(c_{j}^{(\text {in })}\right) .
$$

In (6), we use $L\left(c_{j}^{\text {(in) }}\right)\left[L\left(c_{j}^{\text {(out) })}\right)\right]$ to denote the LDPC decoder input (output). The bit LLRs $L\left(c_{j}\right)$ are determined from symbol LLRs by

$$
L\left(\hat{c}_{j}\right)=\log \frac{\sum_{c: c_{j}=0} \exp [\lambda(S)] \exp \left(\sum_{c: c_{k}=0, k \neq j} L_{a}\left(c_{k}\right)\right)}{\sum_{c: c_{j}=1} \exp [\lambda(S)] \exp \left(\sum_{c: c_{k}=0, k \neq j} L_{a}\left(c_{k}\right)\right)} .
$$




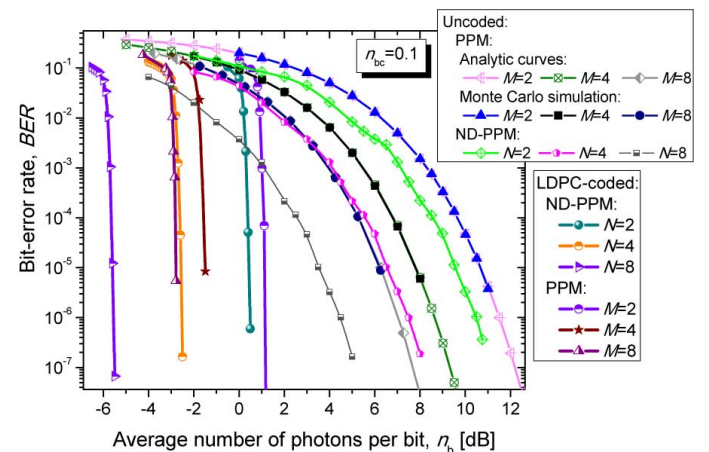

Fig. 2. Uncoded and LDPC(15120, 7560)-coded ND-PPM against PPM

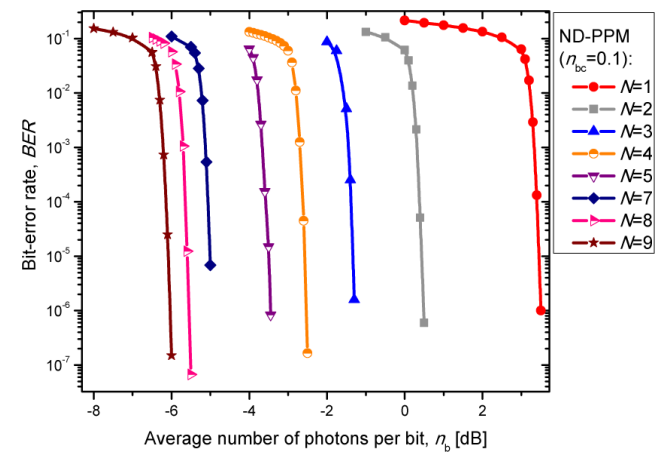

Fig. 3. LDPC(15120, 7560)-coded ND-PPM for different $N$ values.

Such obtained bit LLRs are forwarded to $K$ LDPC decoders operating in parallel, which provide extrinsic bit LLRs for demapper according to (6), and are used as inputs to (5) as the prior information.

\section{Performance Evaluation And Conclusion}

In Fig. 2, we show the BER performance of both uncoded and structured regular (girth-12, column-weight-3) LDPC(15120, 7560)-coded ND-PPM with direct detection versus $M$-ary PPM. The uncoded BER results for PPM obtained by Monte Carlo simulations show excellent agreement with analytic expressions given in [2]. The codeword length is shorter than that in [3], column-weight is set to 3 , while the complexity of regular quasi-cyclic code is lower than that of irregular LDPC code used in [1], whose column-weights range from 2-6 with distribution given in [1]. The quasi-cyclic LDPC code is used in this letter as LDPC codes of different girths, for the same length, have identical complexity (only powers of corresponding permutation matrices are different). On horizontal axis we use average number of photons per bit (in dB-scale) so that ND-PPM and PPM can be compared. The numerical results are independent on bit rate. The data rate dependence can be incorporated by using $n_{b} / T_{s}\left(T_{s}\right.$-the symbol duration) instead of $n_{b}$ on $x$-axis, which requires just a proper scaling of $x$-axis. The results of simulations shown in Figs. 2-5 are obtained for Poisson deep-space optical channel model for the average number of background photons $n_{b c}$ of 0.1 and $L=2$. From Fig. 2 is clear that as we increase the number of dimensions, the improvement of ND-PPM over PPM is larger. The improvement (measured at BER of $10^{-5}$ ) ranges from

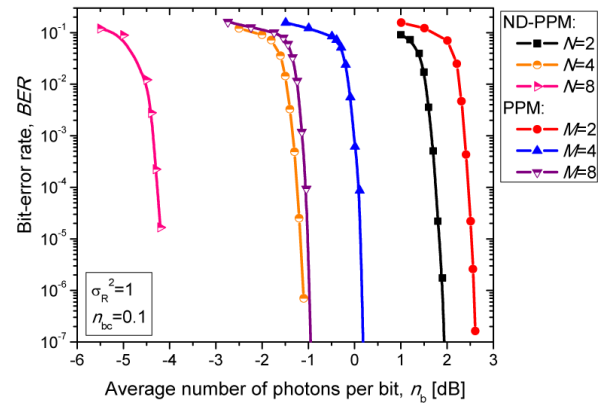

Fig. 4. $\operatorname{LDPC}(15120,7560)$-coded ND-PPM in medium turbulence $\left(\sigma_{R}^{2}=1\right)$.

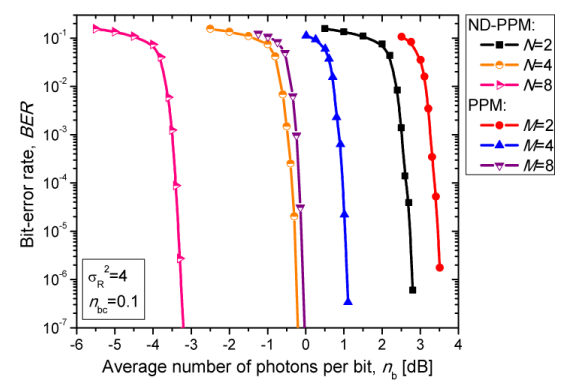

Fig. 5. LDPC $(15120,7560)$-coded ND-PPM in strong turbulence $\left(\sigma_{R}^{2}=4\right)$.

$0.69 \mathrm{~dB}$ for $N=2$ to $2.83 \mathrm{~dB}$ for $N=8$. In Fig. 3, we show LDPC-coded ND-PPM for various dimensions $\left(n_{b c}=0.1\right)$.

In Figs. 4 and 5, we evaluate performance of LDPC-coded ND-PPM in the presence of atmospheric turbulence, under medium (Fig. 4) and strong (Fig. 5) turbulence regimes. For $N=8$, the degradation in performance ranges from $1.46 \mathrm{~dB}$ in medium turbulence regime to $2.25 \mathrm{~dB}$ in strong turbulence regime (at BER of $10^{-5}$ ). The improvement of ND-PPM over PPM is even better in the presence of atmospheric turbulence. In the medium turbulence regime, the improvement of ND-PPM over PPM is $3.19 \mathrm{~dB}$ for $N=8$, while in the strong turbulence regime the improvement is $3.21 \mathrm{~dB}$.

The spectral efficiency of ND-PPM is

$$
\frac{S_{E, N D-P P M}}{S_{E, P P M}}=\frac{\log _{2} L^{N} /\left(N / T_{s}\right)}{\log _{2} M /\left(M / T_{s}\right)}=\frac{M \log _{2} L}{\log _{2} M}
$$

times better than that of PPM. For the same number of dimensions $(M=N)$ and $L=2$, the spectral efficiency of ND-PPM is $N / \log _{2} N$ times better. For example, for $M=N=64$, the spectral efficiency of ND-PPM is 10.667 times better. The spectral efficiency of ND-PPM can further be improved by increasing the number of amplitude levels per coordinate $L$.

\section{REFERENCES}

[1] M. F. Barsoum, B. Moision, M. Fitz, D. Divsalar, and J. Hamkins, "Iterative coded pulse-position-modulation for deep-space optical communications," in Proc. ITW 2007, Lake Tahoe, CA, Sep. 2-6, 2007, pp. 66-71.

[2] S. J. Dolinar, J. Hamkins, B. E. Moision, and V. A. Vilnrotter, "Optical modulation and coding," in Deep Space Optical Communications, H. Hemmati, Ed. Hoboken, NJ: Wiley, 2006.

[3] M. K. Cheng, M. A. Nakashima, B. E. Moision, and J. Hamkins, Optimizations of a Turbo-Like Decoder for Deep-Space Optical Communications IPN Progress Report 42-168, Feb. 15, 2007.

[4] H. Hemmati, "Interplanetary laser communications," Opt. Photon. News, vol. 18, no. 11, pp. 22-27, 2007. 